\title{
Elucidating Energy-Transfer Dynamics Within and Beyond Lanthanide Metal-Organic Frameworks
}

Hui Cheng, ${ }^{a, b}$ Qi Sun, ${ }^{b}$ Shiping Wang, ${ }^{b}$ Yunbo Zhang, ${ }^{a}$ Donghua Fan, ${ }^{a *}$ Jan J.

Huang, ${ }^{a}$ Shengye $\operatorname{Jin}^{b *}$

${ }^{a}$ School of Applied Physics and Materials, Wuyi University, Jiangmen, 529020, China

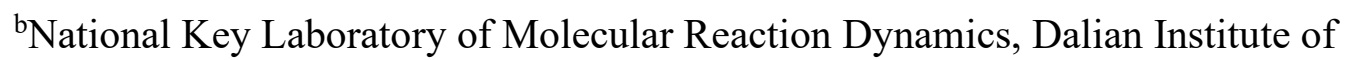

Chemical Physics, Chinese Academy of Sciences, Dalian 116023, China

1. The FT-IR spectra of these Ln-MOFs possess similar characteristic peaks as that of 2,5-dimethoxy-1,4-benzenedicarboxylic acid ( $\left.\mathrm{H}_{2} \mathrm{DMBDC}\right)$, including the absorption peak corresponding to the stretching vibration of carbonyl $(\mathrm{C}=\mathrm{O})$ group at $1690 \mathrm{~cm}^{-1}$, the peaks at $1417 \mathrm{~cm}^{-1}$ representing the methyl $\left(-\mathrm{CH}_{3}\right)$ group,

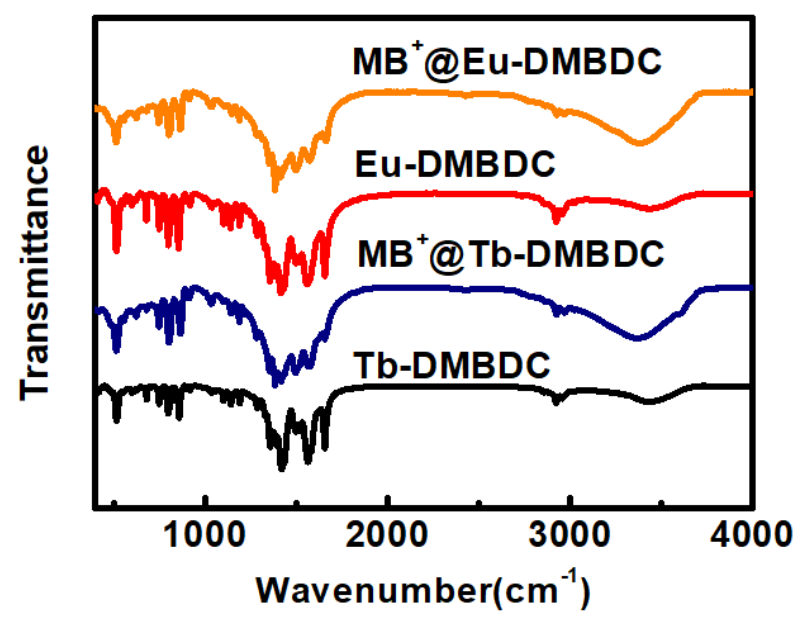

Figure S1. FTIR spectra of $\mathrm{H}_{2} \mathrm{DMBDC}$, Tb-DMBDC, EuDMBDC, MB $@$ Tb-DMBDC, MB $@$ Eu-DMBDC 
as well as the characteristic peaks of $\mathrm{V}_{\mathrm{C}=\mathrm{C}}$ observed at $1560 \mathrm{~cm}^{-1}$. In addition, there are absorption peaks located in $800-900 \mathrm{~cm}^{-1}$, which can be attributed to the formation of the linkage between $\mathrm{Ln}^{3+}$ metal ions and carboxylate of ligand (2,5-dimethoxy-1,4-benzenedicarboxylic acid). These results together suggest the formation of Ln-MOF structure due to coordination of $\mathrm{H}_{2} \mathrm{DMBDC}$ to $\mathrm{Tb} / \mathrm{Eu}$. The broad band at $3600-3000 \mathrm{~cm}^{-1}$ in both lines indicated the presence of $\mathrm{OH}^{-}$ group of water in the samples due to the absorption of water from air. ${ }^{2}$

2. Thermogravimetric (TGA) analyses was performed to evaluate the thermal behavior and thermal stability of these MOFs. As shown in Figure S2, the thermo gravimetric analysis curves of $\mathrm{Eu} / \mathrm{Tb}$-DMBDC is featured by two main steps of weight loss. The first step of weight loss $\left(<430^{\circ} \mathrm{C}\right)$ can be attributed to the loss of guest molecules (water and solvent), which are likely encapsulated in the framework during the synthesis of MOFs. The release of the guest molecules from MOFs at different temperatures can be attributes to the differences of porous state of those MOFs. The second weight loss corresponds to the decomposition of organic linkers. From the results it was found that the thermal stability of MOFs materials was affected by the ligands and linkers.

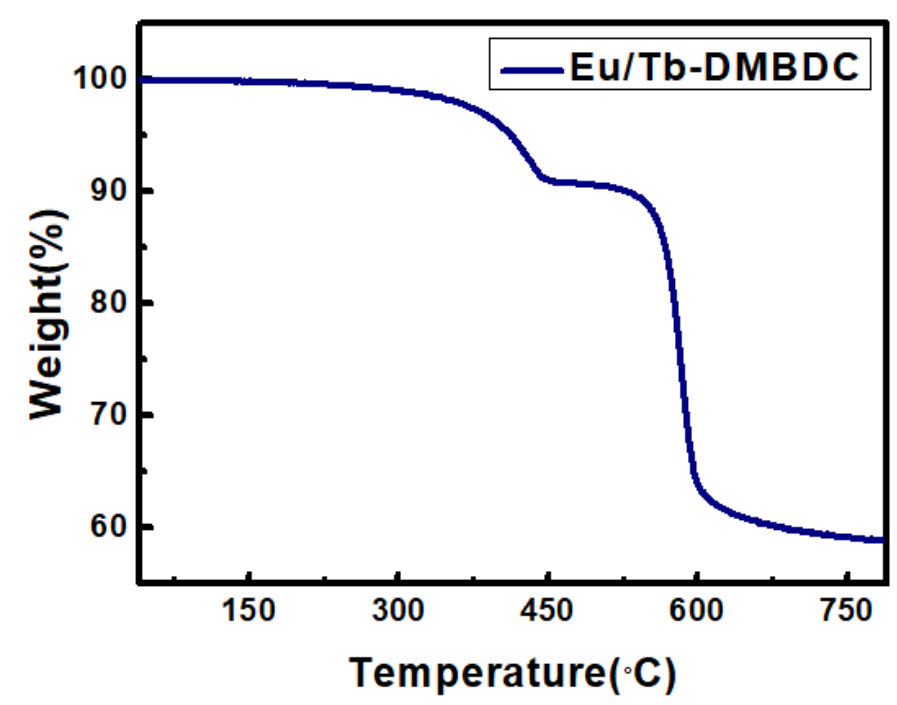

Figure S2. TG spectra of $\mathrm{Eu}_{0.0135} \mathrm{~Tb}_{0.9865-\mathrm{DMBDC}}$ 
3. According to Blasse's formula, the critical distance $\left(\mathrm{R}_{\mathrm{C}}\right)$ between $\mathrm{Tb}^{3+}$ to $\mathrm{Eu}^{3+}$ in $\mathrm{Eu}_{\mathrm{X}} \mathrm{Tb}_{1-\mathrm{X}}-\mathrm{DMBDC}$ can be estimated by:

$$
R_{c}=2\left[\frac{3 V}{4 \pi X_{c} N}\right]^{\frac{1}{3}}
$$

Where $\mathrm{V}$ is the volume and $\mathrm{N}$ is the number of cationic sites of the unit cell, $X_{c}$ is the optimal content of $\mathrm{Tb}^{3+}$ and $\mathrm{Eu}^{3+}$ ions. ${ }^{3-4}$ In the present work, ${ }^{1} \mathrm{~V}$ is $3912.9 \AA^{3}$, and $\mathrm{N}$ is $4, X_{c}$ critical value is 1 . The $\mathrm{R}_{C}$ value calculated is $12.32 \AA$.

4. $\mathrm{N}_{2}$ adsorption experiment

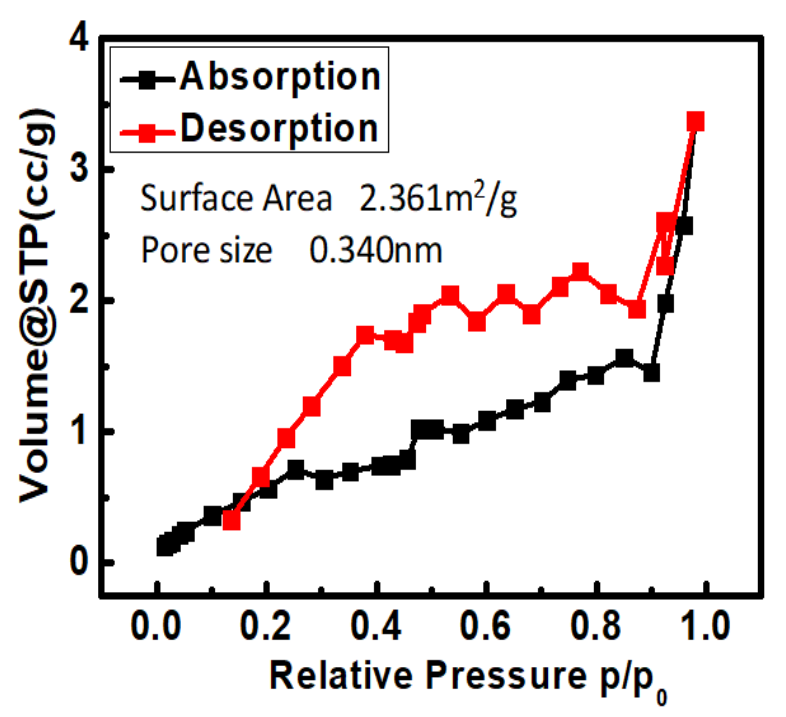

Figure $\mathrm{S} 3 . \mathrm{N}_{2}$ adsorption-desorption isotherm at $77 \mathrm{~K}$ of Tb-DMBDC 


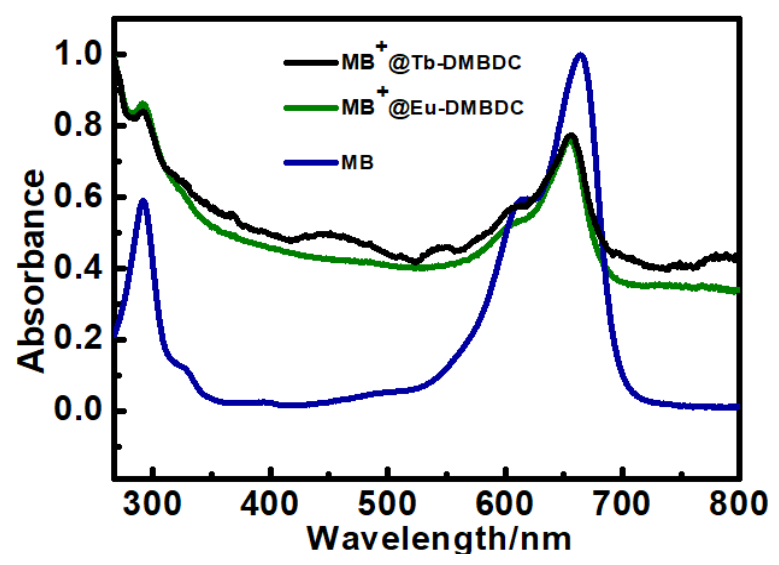

Figure S4. The UV-visible absorption spectrum of $\mathrm{MB}^{+}$, and diffuse reflectance spectra of $\mathrm{MB}^{+} @$ Eu-DMBDC and $\mathrm{MB}^{+} @$ Tb-DMBDC.

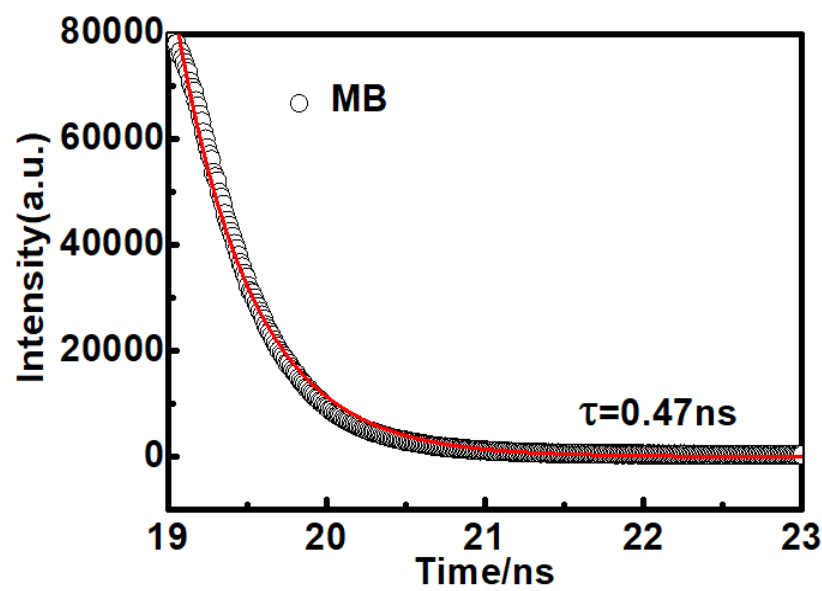

Figure S5 The emission decay curves of MB.

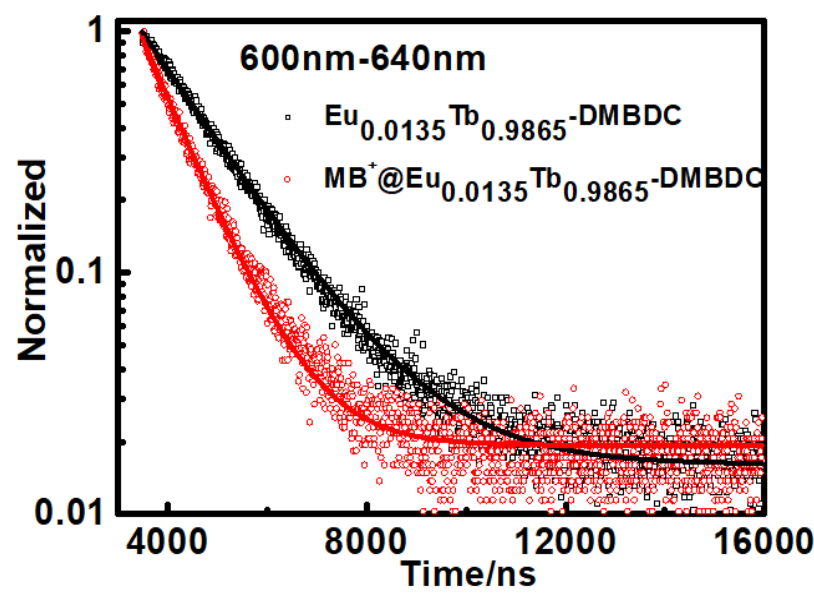

Figure S6. The emission decay curves of $\mathrm{Eu}^{3+}$ ions in $\mathrm{Eu}_{0.0135} \mathrm{~Tb}_{0.9865}$-DMBDC and $\mathrm{MB}^{+} @ \mathrm{Eu}_{0.0135} \mathrm{~Tb}_{0.9865}$-DMBDC. 


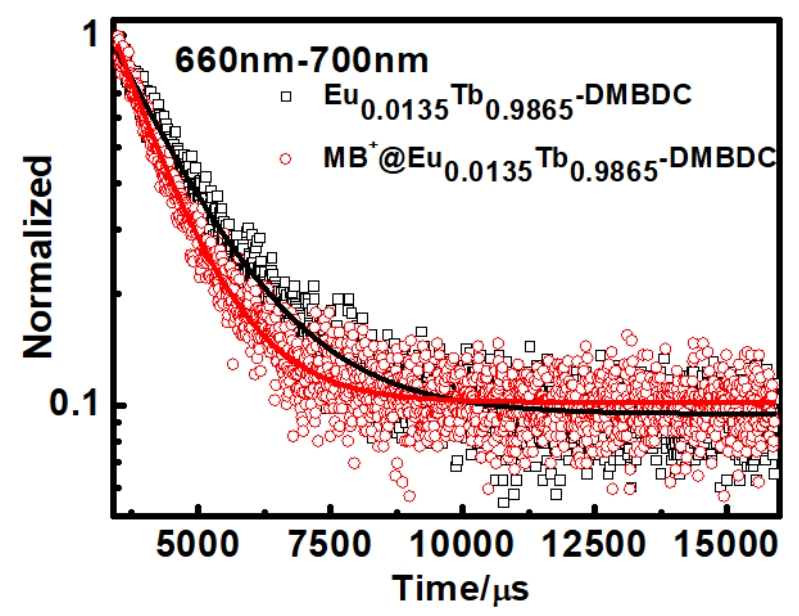

Figure $\mathrm{S} 7$. The emission decay curves in $\mathrm{Eu}_{0.0135} \mathrm{~Tb}_{0.9865}-\mathrm{DMBDC}$ and $\mathrm{MB}^{+} @ \mathrm{Eu}_{0.0135} \mathrm{~Tb}_{0.9865}$-DMBDC monitored at 660 nm-700 nm.

Table S1. ICP-OES for EuxTb1-x-DMBDC

\begin{tabular}{c|c|c|c}
\hline Theoretical value & $\mathrm{Eu}^{3+} \mathrm{mmol} / 1$ & $\mathrm{~Tb}^{3+} \mathrm{mmol} / 1$ & Actual value \\
\hline $\mathrm{Eu}_{0.0045} \mathrm{~Tb}_{0.9955}-\mathrm{DMBDC}$ & 6.273 & 1828.194 & $\mathrm{Eu}_{0.0034} \mathrm{~Tb}_{0.9966}$-DMBDC \\
\hline $\mathrm{Eu}_{0.0135} \mathrm{~Tb}_{0.9865}-\mathrm{DMBDC}$ & 20.296 & 1877.281 & $\mathrm{Eu}_{0.0107} \mathrm{~Tb}_{0.9893}$-DMBDC \\
\hline $\mathrm{Eu}_{0.0225} \mathrm{~Tb}_{0.9775}-\mathrm{DMBDC}$ & 34.920 & 1918.820 & $\mathrm{Eu}_{0.0179} \mathrm{~Tb}_{0.9821}$-DMBDC \\
\hline $\mathrm{Eu}_{0.0315} \mathrm{~Tb}_{0.9685}-\mathrm{DMBDC}$ & 50.355 & 1812.901 & $\mathrm{Eu}_{0.0269} \mathrm{~Tb}_{0.9731}$-DMBDC \\
\hline
\end{tabular}




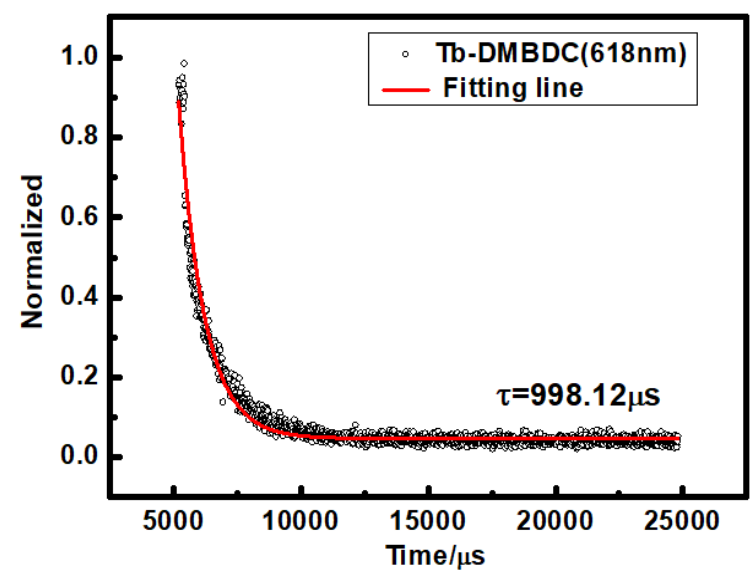

Figure S8. The emission decay curves of $\mathrm{Tb}^{3+}$ ions in Tb-DMBDC monitored at $547 \mathrm{~nm}$. The solid line is the best fits to the experimental result.

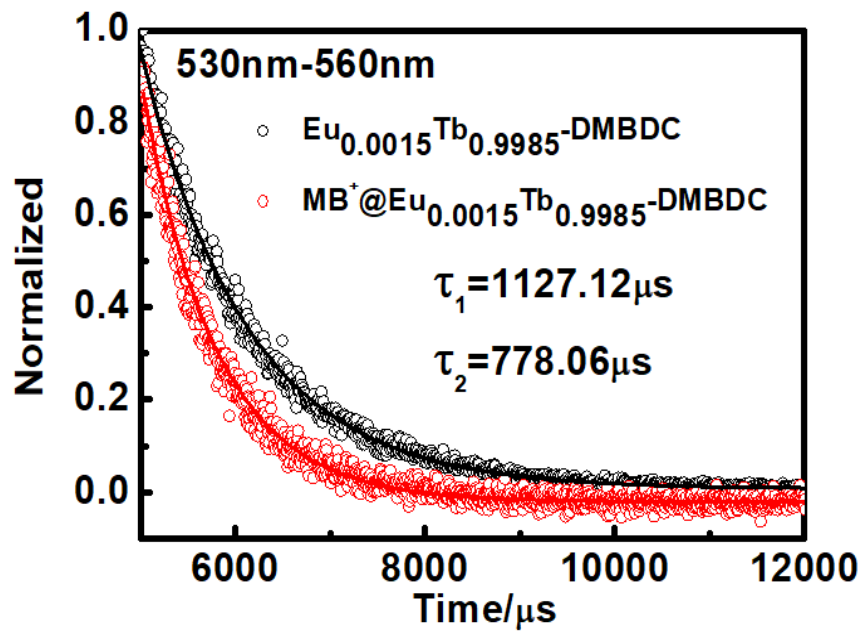

Figure S9. The emission decay curves in $\mathrm{Eu}_{0.0015} \mathrm{~Tb}_{0.9985}$-DMBDC and $\mathrm{MB}^{+} @ \mathrm{Eu}_{0.0015} \mathrm{~Tb}_{0.9985}$-DMBDC monitored at $530 \mathrm{~nm}-560 \mathrm{~nm}$

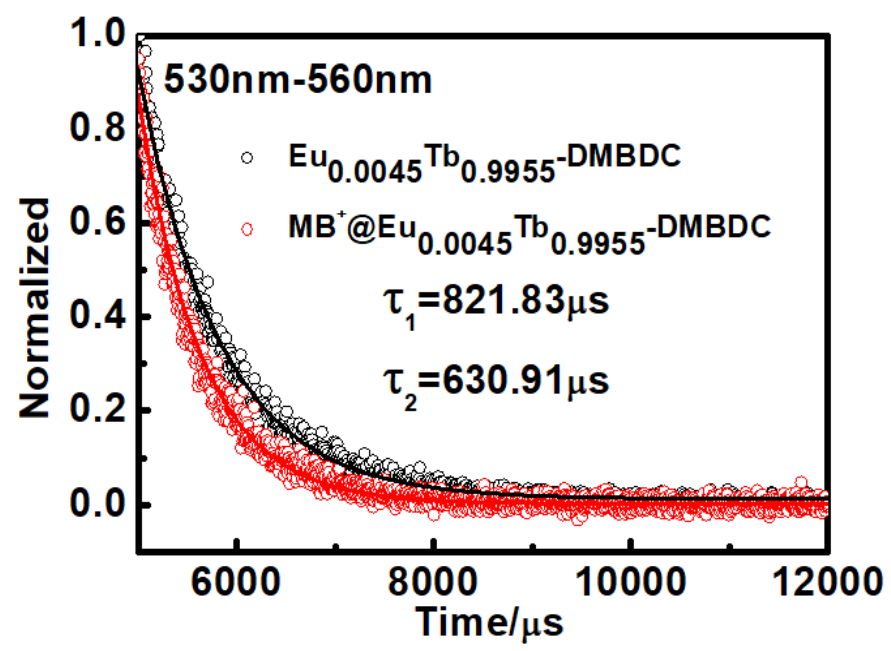

Figure $\mathrm{S} 10$. The emission decay curves in $\mathrm{Eu}_{0.0045} \mathrm{~Tb}_{0.9955}$-DMBDC and $\mathrm{MB}^{+} @ \mathrm{Eu}_{0.0045} \mathrm{~Tb}_{0.9955}$-DMBDC monitored at $530 \mathrm{~nm}-560 \mathrm{~nm}$ 


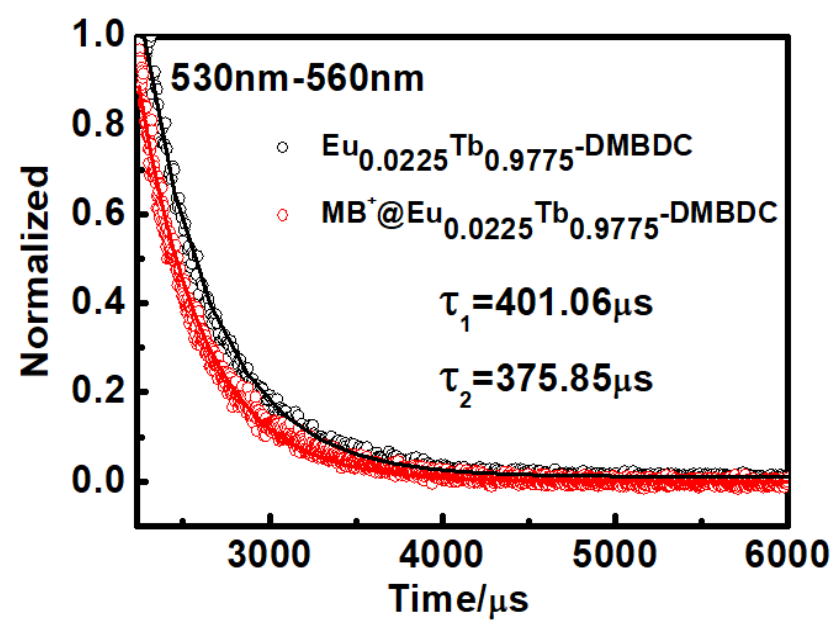

Figure S11. The emission decay curves in $\mathrm{Eu}_{0.0225} \mathrm{~Tb}_{0.9775}$-DMBDC and $\mathrm{MB}^{+} @ \mathrm{Eu}_{0.0225} \mathrm{~Tb}_{0.9775}$-DMBDC monitored at $530 \mathrm{~nm}-560 \mathrm{~nm}$

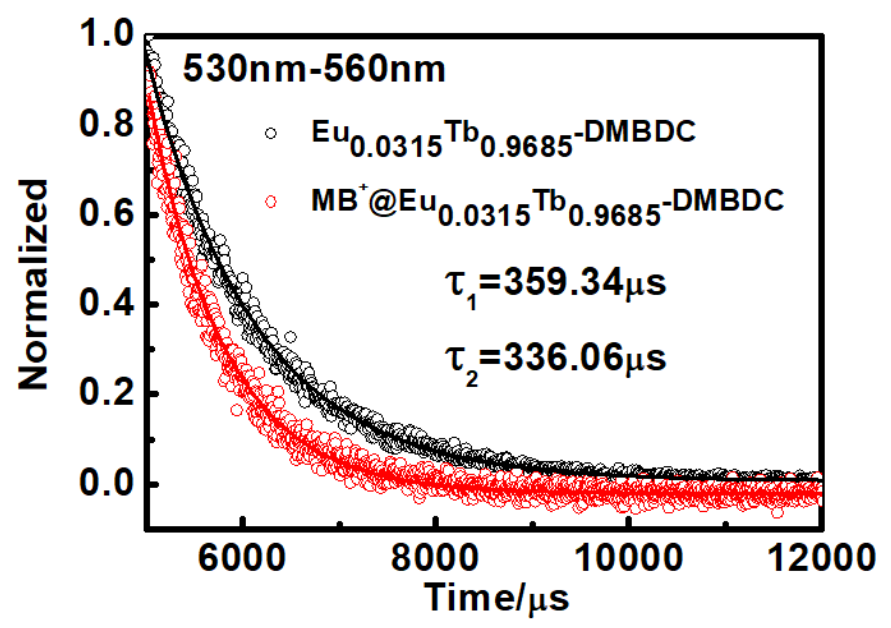

Figure S12. The emission decay curves in $\mathrm{Eu}_{0.0315} \mathrm{~Tb}_{0.9685}$-DMBDC and $\mathrm{MB}^{+} @ \mathrm{Eu}_{0.0315} \mathrm{~Tb}_{0.9685}$-DMBDC monitored at $530 \mathrm{~nm}-560 \mathrm{~nm}$ 
Table S2. The emission lifetimes $(\tau)$ of $\mathrm{Tb}^{3+}$ ions and $\mathrm{Eu}^{3+}$ ions in Ln-DMBDC and $\mathrm{MB}^{+} @$ Ln-DMBDC

\begin{tabular}{c|c|c}
\hline & $\mathrm{Tb}^{3+}(547 \mathrm{~nm})$ & $\mathrm{Eu}^{3+}(613 \mathrm{~nm})$ \\
\hline $\mathrm{Tb}-\mathrm{DMBDC}$ & $1360.17 \mu \mathrm{s}$ & - \\
\hline $\mathrm{Eu}-\mathrm{DMBDC}$ & - & $1215.66 \mu \mathrm{s}$ \\
\hline $\mathrm{Eu}_{0.0015} \mathrm{~Tb}_{0.9985}-\mathrm{DMBDC}$ & $1127.12 \mu \mathrm{s}$ & $1781.58 \mu \mathrm{s}$ \\
\hline $\mathrm{Eu}_{0.0045} \mathrm{~Tb}_{0.9955}-\mathrm{DMBDC}$ & $821.83 \mu \mathrm{s}$ & $1603.72 \mu \mathrm{s}$ \\
\hline $\mathrm{Eu}_{0.0135} \mathrm{~Tb}_{0.9865}-\mathrm{DMBDC}$ & $594.13 \mu \mathrm{s}$ & $1583.90 \mu \mathrm{s}$ \\
\hline $\mathrm{Eu}_{0.0225} \mathrm{~Tb}_{0.9775}-\mathrm{DMBDC}$ & $401.06 \mu \mathrm{s}$ & $1548.77 \mu \mathrm{s}$ \\
\hline $\mathrm{Eu}_{0.0315} \mathrm{~Tb}_{0.9685}-\mathrm{DMBDC}$ & $359.34 \mu \mathrm{s}$ & $1440.58 \mu \mathrm{s}$ \\
\hline $\mathrm{MB}^{+} @ \mathrm{~Tb}_{-} \mathrm{DMBDC}$ & $876.54 \mu \mathrm{s}$ & - \\
\hline $\mathrm{MB}^{+} @ \mathrm{Eu}_{0.0135} \mathrm{~Tb}_{0.9865}-\mathrm{DMBDC}$ & $527.17 \mu \mathrm{s}$ & - \\
\hline
\end{tabular}

Dynamics of FRET from $\mathrm{Ln}^{3+}$ ions to MB molecules.

The $\mathrm{Tb}^{3+}$ excited state decay is described as

$$
\left[T b^{*}\right](t)=A e^{-\left(k_{T b}\right) t}
$$

The $\mathrm{MB}^{+} @ \mathrm{~Tb}^{3+}$ excited state decay is described as

$$
\begin{gathered}
{\left[M B^{+} @ T b^{*}\right](t)=A_{1} e^{-\left(k_{T b}+k_{M B 1}\right) t}} \\
k_{M B 1}=\frac{1}{\tau_{M B^{+}} @ T b}-\frac{1}{\tau_{T b}}
\end{gathered}
$$

According to the Table S2, the value of $k_{M B 1}$ is $4.06 \mathrm{~ms}^{-1}, \tau_{M B 1}$ is $2465.17 \mu \mathrm{s}^{-1}$.

$\mathrm{The} \mathrm{Eu}^{3+} / \mathrm{Tb}^{3+}$ excited state decay is described as

$$
\left[E u / T b^{*}\right](t)=A_{3} e^{-\left(k_{E u / T b}\right) t}
$$

The $\mathrm{MB}^{+} @ \mathrm{Eu}^{3+} / \mathrm{Tb}^{3+}$ excited state decay is described as

$$
\begin{gathered}
{\left[M B^{+} @ E u / T b^{*}\right](t)=A_{4} e^{-\left(k_{E u / T b}+k_{M B 2}\right) t}} \\
k_{M B 2}=\frac{1}{\tau_{M B^{+} @ E u / T b}}-\frac{1}{\tau_{E u / T b}}
\end{gathered}
$$

According to the Table S2, the value of $k_{M B 2}$ is $2.1 \mathrm{~ms}^{-1}, \tau_{M B 2}$ is $4677.48 \mu \mathrm{s}^{-1}$.

$\kappa_{\mathrm{MB}}$ is the energy transfer rate from $\mathrm{Ln}^{3+}$ ions to $\mathrm{MB}^{+}$molecule. 
Table S3. The energy transfer rate $\left(\kappa_{E N T}\right)$ and energy transfer efficiency $\left(\eta_{E N T}\right)$ for $\mathrm{EuxTb}_{1-\mathrm{x}-\mathrm{DMBDC}}$

\begin{tabular}{c|c|c}
\hline & $\kappa_{E N T}$ & $\eta_{E N T}$ \\
\hline $\mathrm{Eu}_{0.0015} \mathrm{~Tb}_{0.9985}-\mathrm{DMBDC}$ & $0.152 \mathrm{~ms}^{-1}$ & $17.13 \%$ \\
\hline $\mathrm{Eu}_{0.0045} \mathrm{~Tb}_{0.9955}-\mathrm{DMBDC}$ & $0.438 \mathrm{~ms}^{-1}$ & $39.58 \%$ \\
\hline $\mathrm{Eu}_{0.0135} \mathrm{~Tb}_{0.9865}-\mathrm{DMBDC}$ & $0.948 \mathrm{~ms}^{-1}$ & $56.32 \%$ \\
\hline $\mathrm{Eu}_{0.0225} \mathrm{~Tb}_{0.9775}-\mathrm{DMBDC}$ & $1.758 \mathrm{~ms}^{-1}$ & $70.52 \%$ \\
\hline $\mathrm{Eu}_{0.0315} \mathrm{~Tb}_{0.9685}-\mathrm{DMBDC}$ & $2.050 \mathrm{~ms}^{-1}$ & $73.58 \%$ \\
\hline
\end{tabular}

Table S4. The energy transfer efficiency $\left(\eta_{E N T}\right)$ and energy transfer time $\left(t_{E N T}\right)$ from $\mathrm{Tb}^{3+}$ to $\mathrm{MB}^{+}$for $\mathrm{MB}^{+} @ \mathrm{Eu}_{\mathrm{X}} \mathrm{Tb}_{1-\mathrm{X}}-\mathrm{DMBDC}$

\begin{tabular}{c|l|l|l|l|l}
\hline & $\begin{array}{l}\mathrm{Eu}_{0.0015} \\
\mathrm{~Tb}_{0.9985^{-}} \\
\mathrm{DMBDC}\end{array}$ & $\begin{array}{l}\mathrm{Eu}_{0.0045} \\
\mathrm{~Tb}_{0.9955^{-}} \\
\mathrm{DMBDC}\end{array}$ & $\begin{array}{l}\mathrm{Eu}_{0.0135} \\
\mathrm{~Tb}_{0.9865^{-}} \\
\mathrm{DMBDC}\end{array}$ & $\begin{array}{l}\mathrm{Eu}_{0.0225} \\
\mathrm{~Tb}_{0.9775^{-}} \\
\mathrm{DMBDC}\end{array}$ & $\begin{array}{l}\mathrm{Eu}_{0.0315} \\
\mathrm{~Tb}_{0.9685} \\
\mathrm{DMBDC}\end{array}$ \\
\hline $\begin{array}{c}\tau_{1} \\
(\mathrm{Eu} / \mathrm{Tb}-\mathrm{DMBDC})\end{array}$ & $1127.12 \mu \mathrm{s}$ & $821.83 \mu \mathrm{s}$ & $594.13 \mu \mathrm{s}$ & $401.06 \mu \mathrm{s}$ & $359.34 \mu \mathrm{s}$ \\
\hline $\begin{array}{c}\tau_{2} \\
\mathrm{MB} @ \mathrm{Eu} / \mathrm{Tb}-\end{array}$ & $778.06 \mu \mathrm{s}$ & $630.91 \mu \mathrm{s}$ & $527.17 \mu \mathrm{s}$ & $375.85 \mu \mathrm{s}$ & $336.06 \mu \mathrm{s}$ \\
\hline$\eta_{E N T}$ & $30.97 \%$ & $23.23 \%$ & $11.27 \%$ & $6.29 \%$ & $6.48 \%$ \\
\hline$t_{E N T}$ & $2512.37 \mu \mathrm{s}$ & $2715.84 \mu \mathrm{s}$ & $4677.48 \mu \mathrm{s}$ & $5979.07 \mu \mathrm{s}$ & $5187.26 \mu \mathrm{s}$ \\
\hline
\end{tabular}

(1) Cui, Y.; Xu, H.; Yue, Y.; Guo, Z.; Yu, J.; Chen, Z.; Gao, J.; Yang, Y.; Qian, G.; Chen, B., A Luminescent Mixed-Lanthanide Metal-Organic Framework Thermometer. J. Am. Chem. Soc. 2012, 134, 3979-3982.

(2) Lucarelli, C.; Galli, S.; Maspero, A.; Cimino, A.; Bandinelli, C.; Lolli, A.; Velasquez Ochoa, J.; Vaccari, A.; Cavani, F.; Albonetti, S., Adsorbent-Adsorbate Interactions in the Oxidation of HMF Catalyzed by Ni-Based MOFs: A DRIFT and FT-IR Insight. $J$. Phys. Chem. C 2016, 120, 15310-15321.

(3) Zhang, J.; He, Y.; Qiu, Z.; Zhang, W.; Zhou, W.; Yu, L.; Lian, S., Site-Sensitive Energy Transfer Modes in $\mathrm{Ca}_{3} \mathrm{Al}_{2} \mathrm{O}_{6}: \mathrm{Ce}^{3+} / \mathrm{Tb}^{3+} / \mathrm{Mn}^{2+}$ Phosphors. Dalton. Trans. 2014, 
$43,18134-18145$

(4) Wang, H.; Chen, X.; Teng, L.; Xu, D.; Chen, W.; Wei, R.; Hu, F.; Sun, X.; Guo, H., Adjustable Emission and Energy Transfer Process in $\mathrm{BaGd}_{2} \mathrm{O}_{4}: \mathrm{Bi}^{3+}, \mathrm{Eu}^{3+}$ Phosphors. $J$. Lumin. 2019, 206, 185-191. 